160414 RSS Book Review Final - Christian Hennig - Handbook of Cluster A...

RSS Book Review: Ed. Christian Hennig et al. "Handbook of Cluster Analysis"

Title: Handbook of Cluster Analysis; Ed. Christian Hennig, Marina Meila, Fionn Murtagh \& Roberto Rocci; 2016; CRC Press, taylor \& Francis Group, 6000 Broken Sound Parkway NW, Suite 300, Boca Raton, FL 33487-2742; 753 pp; Hardback f61.59, eBook f53.89; ISBN-13: 978-1-4665-5188-6.

\title{
400 words.
}

From the wide-ranging Handbooks of Modern Statistical Methods series, this book seeks to be a non-exhaustive guide to the subject in a large ( $>400 \mathrm{k}$ documents in the literature) $\&$ expanding field. The book is well laid out over 31 chapters each having its own introduction \& conclusion, spanning the material in a logical manner aiding accessibility. Its main focus is on partitioning sets, and care is taken to explain the exploratory nature of the analysis in contrast to the predictive task of classification (i.e. supervised).

Although the book concerns unsupervised clustering, the authors reiterate the importance of input from the researcher in suitable choice of models as "different algorithms often yield strikingly different clusterings from the same dataset". Starting with a history of the field, they layout a consistent approach to collating the different techniques from different disciplines as well as ties between fields (e.g. linkage cluster methods \& ties to graph theory). This broadly moves to metricbased methods (similarity/dissimilarity measures) \& then to probability models and other techniques (e.g. semi-supervised). The last chapters address cluster validation, while the longer final chapter consolidates the book by addressing the strategic choices needed to select an appropriate clustering technique (i.e. pre-processing data, or relating the method to clustering aims).

Big data issues are explored, leading to dimension reduction \& variable selection considerations such as PCA or alternative aggregation techniques -then linking to the choice of suitable visualisations which is explicitly given its own chapter. Many different types of data have been used in the examples (e.g. genetics), and where colour plots are crucial links from this B\&W book to websites have been provided.

The more recent topic of network analysis is illustrated with clear examples (e.g. a mixed membership stochastic block model), and references are given to sources of code/software, although code is not included with the examples. Cluster validation techniques such as the Silhoutte Criterion are explained as they become relevant (leading to how they can be extended to fuzzy clustering models) rather than linked till later in the book $\&$ the chapters on validation.

This is a comprehensive reference guide, well organised \& has an approachable style with many examples. Great care has been taken to provide an appropriate level of detail using illustrative \& topical examples. As an overview of an increasingly important field, it provides a vital first reference guide for a range of techniques \& modelling considerations.

Mark Pilling

Manchester 